\title{
Analysis and Treatment of Complications after Unicompartmental Knee Arthroplasty
}

\author{
Kyung Tae Kim, MD, Song Lee, MD, Jae Il Lee, MD, and Jin Woo Kim, MD
}

Department of Orthopedic Surgery, Seoul Sacred Heart General Hospital, Seoul, Korea

Purpose: To analyze the causes and types of complications after unicompartmental knee arthroplasty (UKA) and determine proper prevention and treatment methods.

Materials and Methods: A total of 1,576 UKAs were performed for osteoarthritis of the knee from January 2002 to December 2014 at one institution. We analyzed complications after UKA retrospectively and investigated proper methods of treatment.

Results: A total of 89 complications (5.6\%) occurred after UKA. Regarding the type of complications after UKA, there were 42 cases of dislocation of the mobile bearing, 23 cases of loosening of the prosthesis, 6 cases of periprosthetic fracture, 3 cases of polyethylene wear, 3 cases of progression of arthritis in the contralateral compartment, 2 cases of medial collateral ligament injury, 2 cases of impingement, 5 cases of infection, 1 case of arthrofibrosis, and 2 cases of failure due to unexplained pain. The most common complication after UKA was mobile bearing dislocation in the mobile-bearing knees and loosening of the prosthesis in the fixed-bearing knees, but polyethylene wear and progression of arthritis were relatively rare. The complications were treated with conversion to total knee arthroplasty in 58 cases and simple bearing change in 21 cases.

Conclusions: The most common complication after UKA was dislocation of the mobile bearing. When a complication occurs after UKA, appropriate treatment should be performed after accurate analysis of the cause of complication.

\section{Keywords: Knee, Osteoarthritis, Unicompartmental arthroplasty, Complication}

\section{Introduction}

Unicompartmental knee arthroplasty (UKA) is a surgical intervention for degenerative arthritis of the knee that has been frequently employed since 1970s. Initially, the procedure was often associated with poor outcomes; however, recent improvements in implant design and surgical technique have contributed to the evolution of the technique so that it is currently performed at an increasing frequency producing satisfactory results ${ }^{1,2}$. The effi-

Received June 18, 2015; Revised (1st) September 4, 2015;

(2nd) October 19, 2015; (3rd) October 20, 2015;

Accepted November 3, 2015

Correspondence to: Kyung Tae Kim, MD

Department of Orthopedic Surgery, Seoul Sacred Heart General Hospital, 259 Wangsan-ro, Dongdaemun-gu, Seoul 02488, Korea Tel: +82-2-966-1616, Fax: +82-2-968-2394

E-mail: kktkimos@hanmail.net

This is an Open Access article distributed under the terms of the Creative Commons Attribution Non-Commercial License (http://creativecommons.org/licenses/by-nc/4.0/) which permits unrestricted non-commercial use, distribution, and reproduction in any medium, provided the original work is properly cited. cacy of UKA still remains controversial due to disparate interpretations of the mid- and long-term outcomes. UKA is beneficial in that it minimizes loss of knee function and promotes rapid recovery due to the need for a small skin incision and minimal bone resection during surgery. Accordingly, the mortality and morbidity rates from early complications after UKA are relatively low. In the long-term, however, the procedure yields higher revision rates due to complications than total knee arthroplasty (TKA) ${ }^{3-7)}$.

Complications following UKA have distinctive characteristics, and consensus on the causes and proper treatment methods has been elusive ${ }^{8,9)}$. Although various studies have described complications after UKA, they are mostly based on a small number of cases ${ }^{3,410-13)}$. In addition, the incidence and type of complications are different from study to study depending on the type and design of implant, follow-up period, and the surgeon who performed the surgery. In this study, we analyzed complications that occurred after UKA to elucidate the causes, types, and optimal prevention and treatment measures in a relatively large number of patients (1,331 patients; 1,576 knees) for a single-institution study. 


\section{Materials and Methods}

\section{Patients}

Between January 2002 and December 2014, 1,576 UKAs were performed on 1,331 patients by two authors of this study (Kim $\mathrm{KT}$ and Lee S) at our institution. The surgery was carried out in a minimally invasive manner in all knees and in the medial compartment of the knee in most cases $(n=1,552)$. Ambulation was allowed immediately after surgery. From the 3 rd postoperative day, after wound care and dressing removal, controlled exercises using a continuous passive motion machine and active motion exercises were initiated.

The implant used for UKA was Oxford mobile-bearing prosthesis (Biomet, Warsaw, IN, USA) in 1,410 knees, Preservation mobile-bearing prosthesis (Depuy, Warsaw, IN, USA) in 31knees, Advance fixed-bearing prosthesis (Wright, Arlington, TN, USA) in 22 knees, Preservation fixed-bearing prosthesis (Depuy) in 14 knees, ZUK fixed-bearing prosthesis (Zimmer, Warsaw, IN, USA) in 94 knees, Triathlon fixed-bearing prosthesis (Howmedica Osteonics, Allendale, NJ, USA) in 4 knees, and HLS fixed-bearing prosthesis (Tornier Inc., Grenoble, France) in $1 \mathrm{knee.} \mathrm{Of} \mathrm{the} \mathrm{total}$ 1,576 UKAs, 1,425 were performed on females and 151 on males. The mean age of the patients was 62 years (range, 43 to 86 years). The mean follow-up period of the patients was 7 years and 10 months (range, 6 months to 14 years and 5 months) (Table 1).

The mean follow-up period was evaluated according to the

Table 1. Demographics

\begin{tabular}{lcc}
\hline \multicolumn{1}{c}{ Parameter } & UKA & Complication \\
\hline Total & 1,576 & 89 \\
Gender (F:M) & $1,425: 151$ & $86: 3$ \\
Medial:lateral & $1,552: 24$ & $89: 0$ \\
Age (yr), mean (range) & $62(43-86)$ & $66(46-82)$ \\
No. of implants & & \\
Oxford mobile & 1,410 & 79 \\
Preservation mobile & 31 & 3 \\
Preservation fixed & 14 & 1 \\
Advance fixed & 22 & 5 \\
HLS fixed & 1 & 1 \\
ZUK fixed & 94 & 0 \\
Triathlon fixed & 4 & 0 \\
Follow-up period (mo), mean (range) & $94(6-168)$ & \\
Time to reoperation (mo), mean (range) & & $54(0-144)$ \\
\hline
\end{tabular}

UKA: unicompartmental knee arthroplasty. implant design: Oxford mobile-bearing prosthesis, 7 years and 2 months (range, 6 months to 14 years and 5 months); Preservation mobile-bearing prosthesis, 11 years and 8 months (range, 11 years and 1 month to 12 years and 4 months); Advance fixedbearing prosthesis, 9 years and 2 months (range, 9 years to 9 years and 4 months); Preservation fixed-bearing prosthesis, 12 years and 6 months (range, 12 years and 4 months to 12 years and 10 months); ZUK fixed-bearing prosthesis, 1 year and 8 months (range, 6 months to 2 years and 7 months); Triathlon fixed-bearing prosthesis, 7 months (range, 6 to 8 months); and HLS fixedbearing prosthesis, 6 months.

\section{Indications and Preoperative Diagnosis}

The surgery was performed in patients with pain in one compartment of the knee and limited range of motion (ROM) without inflammatory arthropathy of the knee and abnormality of the anterior/posterior cruciate ligaments. The preoperative ROM was at least $110^{\circ}$ with less than $15^{\circ}$ of varus deformity and $15^{\circ}$ of flexion contracture. Radiographic evidence of femorotibial joint arthritis unaccompanied by pain was also considered as an indication for the surgery. Other indications for UKA include unicompartmental osteoarthritis, primary osteonecrosis or osteochondritis dissecans, and traumatic arthritis. The preoperative diagnosis was degenerative arthritis of the knee in all patients in this study.

\section{Assessments and Analyses}

Preoperative medical records and postoperative follow-up data were reviewed for analysis. Follow-up was conducted on a regular basis at 3 months, 6 months, and 12 months after surgery and then annually for clinical and radiological assessments, and postoperative complications were retrospectively investigated. For clinical assessment, knee pain, ROM, and Knee Society knee score and function score were evaluated using the Knee Society Clinical Rating System. For radiological assessment, weightbearing radiographs of the knee were obtained to evaluate the femorotibial angle, bone change, component loosening, wear, deformity or dislocation, and progression of arthritis to the contralateral compartment.

In patients with complications after UKA, timing of complications during follow-up was recorded and the cause of complication was investigated during revision surgery. The methods used for the treatment of complications were analyzed. In addition, the incidence and type of complications were compared according to gender, age, medial/lateral compartment, and implant design and type. 


\section{Results}

A total of 1,576 UKAs were performed between January 2002 and December 2014 at our institution. Postoperative complications occurred in 89 knees (83 patients, 5.6\%), and 86 of them were found in females and 3 in males. Their mean age was 61 years (range, 46 to 81 years) at the time of initial UKA and 66 years (range, 46 to 82 years) at the time of revision surgery (Table 1). Complications were observed only in the knees with medial UKA.

The causes of UKA failure include bearing dislocation $(n=42)$, component loosening $(n=23,11$ cases of femoral component loosening, 8 cases of tibial component loosening, and 4 cases of both femoral and tibial component loosening), periprosthetic fracture $(n=6)$, polyethylene wear/ destruction $(n=3)$, progression of arthritis to the other compartment $(n=3)$, medial collateral ligament (MCL) injury $(n=2)$, impingement $(n=2)$, infection $(\mathrm{n}=5)$, ankylosis $(\mathrm{n}=1)$, and unexplained pain $(\mathrm{n}=2)$.

\section{Complications according to Implant Design}

The Oxford mobile-bearing prosthesis was used in 1,410 knees and 79 cases $(5.6 \%)$ of failure occurred due to bearing dislocation in 42 knees, component loosening in 15 knees, polyethylene wear/destruction in 3 knees, progression of arthritis to the other compartment in 3 knees, MCL injury in 2 knees, periprosthetic fracture in 4 knees, impingement in 2 knees, infection in 5 knees, ankylosis in 1 knee, and unexplained pain in 2 knees. The Preservation mobile-bearing prosthesis $(n=31)$ resulted in 3 cases $(9.7 \%)$ of failure related to tibial component loosening $(n=2)$ and medial tibial condylar fracture $(n=1)$. In the knees with the Advance fixed-bearing prosthesis $(n=22)$, there were 5 cases $(23 \%)$ of complications resulting from femoral component loosening $(n=2)$, tibial component loosening $(n=2)$, and medial tibial condylar fracture $(n=1)$. Component loosening was the cause of failure in $1(7.1 \%)$ of the 14 knees with the Preservation fixed-bearing prosthesis and in the only knee with the HLS fixed-bearing prosthesis. In the knees with the ZUK prosthesis and Triathlon fixedbearing prosthesis, none of the complications was noted (Table 2).

\section{Complications according to Implant Type}

\section{1) Mobile-bearing type}

Mobile-bearing type prostheses were used in 1,441 knees and complications occurred in $82(5.7 \%)$ of them . The most prevalent complication was bearing dislocation $(n=42,2.9 \%)$, followed by component loosening $(n=17,1.2 \%)$. The component loosening was observed only in the femur in 9 knees $(0.6 \%)$, only in the tibia in 4 knees $(0.3 \%)$, and both in the femur and tibia in 4 knees

Table 2. Complications after Unicompartmental Knee Arthroplasty

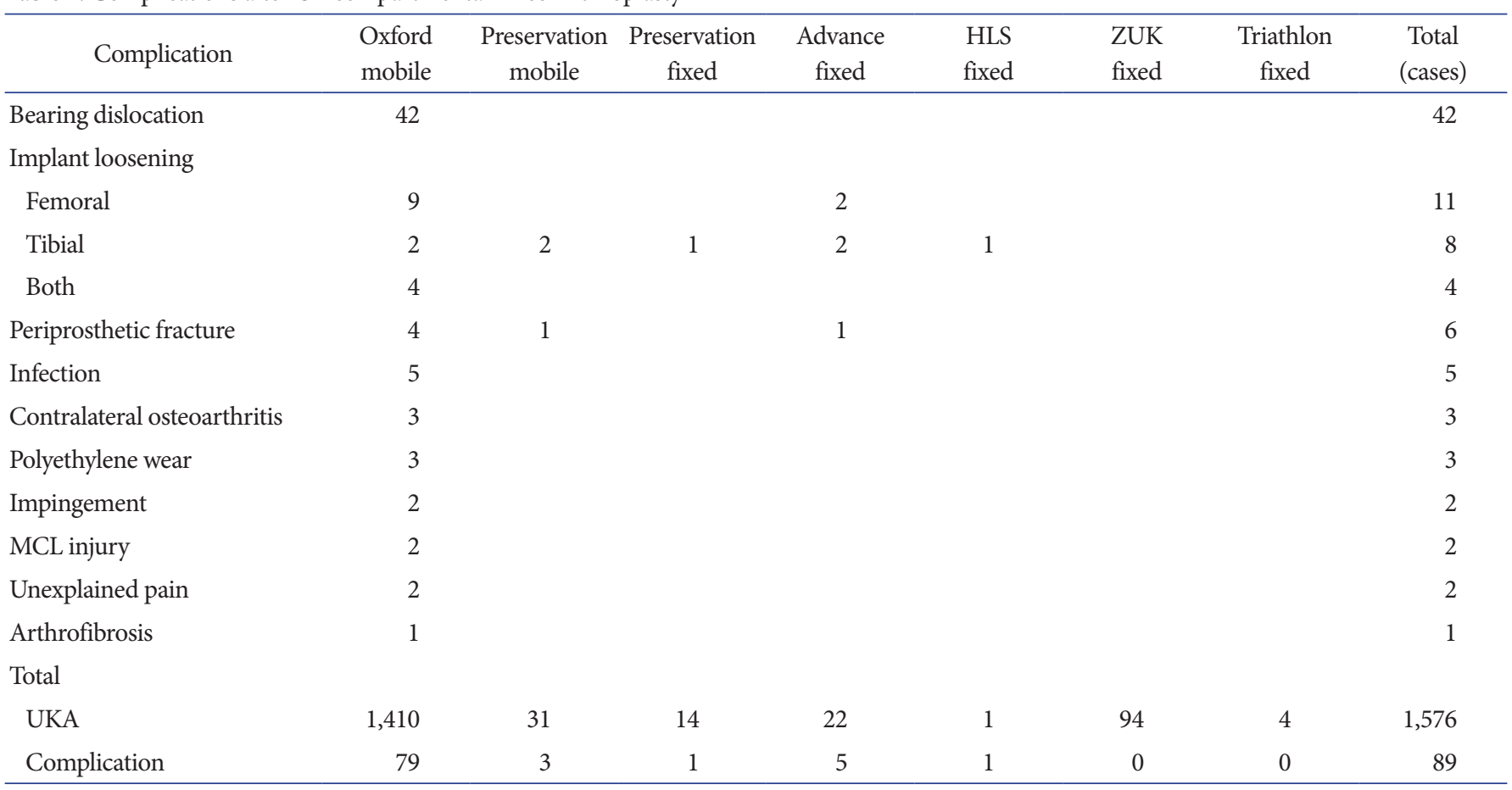

MCL: medial collateral ligament, UKA: unicompartmental knee arthroplasty. 
(0.3\%). Other causes of failure include polyethylene wear/destruction ( $n=3,0.3 \%)$, progression of arthritis to the other compartment $(n=3,0.3 \%)$, MCL injury $(n=2,0.2 \%)$, periprosthetic fracture $(n=5,0.4 \%)$, impingement $(n=2,0.2 \%)$, infection $(n=5$, $0.4 \%)$, ankylosis $(n=1,0.1 \%)$, and unexplained pain $(n=2,0.2 \%)$.

Of the total mobile-bearing type prostheses, the meniscalbearing Oxford prosthesis resulted in dislocation of bearing in 42 knees (3.0\%), component loosening in 15 knees (1.1\%). In the knees with the tracked-bearing Preservation prosthesis, bearing dislocation was not observed, but tibial component loosening occurred in 2 knees $(6.6 \%)$ and medial tibial condylar fracture in 1 knee $(3.3 \%)$ (Table 2$)$.

\section{2) Fixed-bearing type}

Complications were found in 7 (5.1\%) of the 135 knees with the fixed-bearing type prostheses: component loosening in 6 knees (4.4\%) and medial tibial condylar fracture in 1 knee. In the knees with the all-polyethylene fixed-bearing type prostheses (Advance, Preservation, and HLS prostheses), there were 6 cases (16\%) of component loosening and 1 case $(2.7 \%)$ of medial tibial condylar fracture. In the knees with the metal-backed fixed-bearing type prostheses (ZUK and Triathlon prostheses), none of the complications was noted (Table 2).

\section{Complications according to Gender}

In the 151 male knees, 3 cases (2.0\%) of complications were observed: bearing dislocation $(n=2)$ and femoral component loosening $(n=1)$. In the 1,425 female knees, 86 cases $(6.0 \%)$ of complication were noted.

\section{Complications according to Medial/Lateral UKA}

All the complications were found in the knees with medial UKA (5.7\%). None of the complications was observed in the knees with lateral UKA.

\section{Timing of Complications}

The mean interval from UKA to the occurrence of complications was 4 years and 6 months (range, 0 [during operation] to 12 years). Complications in the knees with mobile-bearing prostheses were observed at a mean of 4 years and 4 months (range, 3 months to 11 years and 6 months) after surgery for bearing dislocation, 4 years and 10 months (range, 4 months to 11 years and 9 months) after surgery for component loosening. 8 years and 4 months (range, 6 years and 7 months to 10 years and 9 months) after surgery for polyethylene wear/destruction, 11 years and 6 months (range, 11 years and 10 months to 12 years) after surgery for progression of arthritis to the contralateral compartment, 1 year and 7 months (range, 0 [during operation] to 5 years and 7 months) after surgery for periprosthetic fracture, and 5 years and 3 months (range, 1 year and 5 months to 10 years and 10 months) after surgery for infection (Table 3).

\section{Treatment of Complications}

Complications were found in 89 knees following UKA. Of those, 58 knees were treated with conversion TKA, 1 with revision UKA, and 21 with simple bearing change. Complications in the remaining knees were treated with arthroscopic management $(n=2)$, open reduction and internal fixation $(n=3)$, closed reduction and internal fixation $(n=1)$, manipulation $(n=1)$, and

Table 3. Time Interval between Unicompartmental Knee Arthroplasty and Complications

\begin{tabular}{|c|c|c|c|c|c|c|}
\hline Complication (mo) & Oxford mobile & Preservation mobile & Preservation fixed & Advance fixed & HLS fixed & Total \\
\hline Bearing dislocation & $51.9 \pm 44.0$ & & & & & $51.9 \pm 44.0$ \\
\hline Implant loosening & $73.9 \pm 44.4$ & $57.5 \pm 13.4$ & $66 \pm 0.0$ & $11.7 \pm 8.3$ & $4.0 \pm 0.0$ & $58.2 \pm 44.5$ \\
\hline Infection & $63.0 \pm 41.6$ & & & & & $63.0 \pm 41.6$ \\
\hline Contralateral osteoarthritis & $138.0 \pm 7.2$ & & & & & $138.0 \pm 7.2$ \\
\hline Impingement & $15.0 \pm 8.5$ & & & & & $15.0 \pm 8.5$ \\
\hline MCL injury & $19.0 \pm 22.0$ & & & & & $19.0 \pm 22.0$ \\
\hline Unexplained pain & $16.0 \pm 7.1$ & & & & & $16.0 \pm 7.1$ \\
\hline Arthrofibrosis & $1.0 \pm 0.0$ & & & & & $1.0 \pm 0.0$ \\
\hline
\end{tabular}

Values are presented as mean \pm standard deviation.

MCL: medial collateral ligament. 

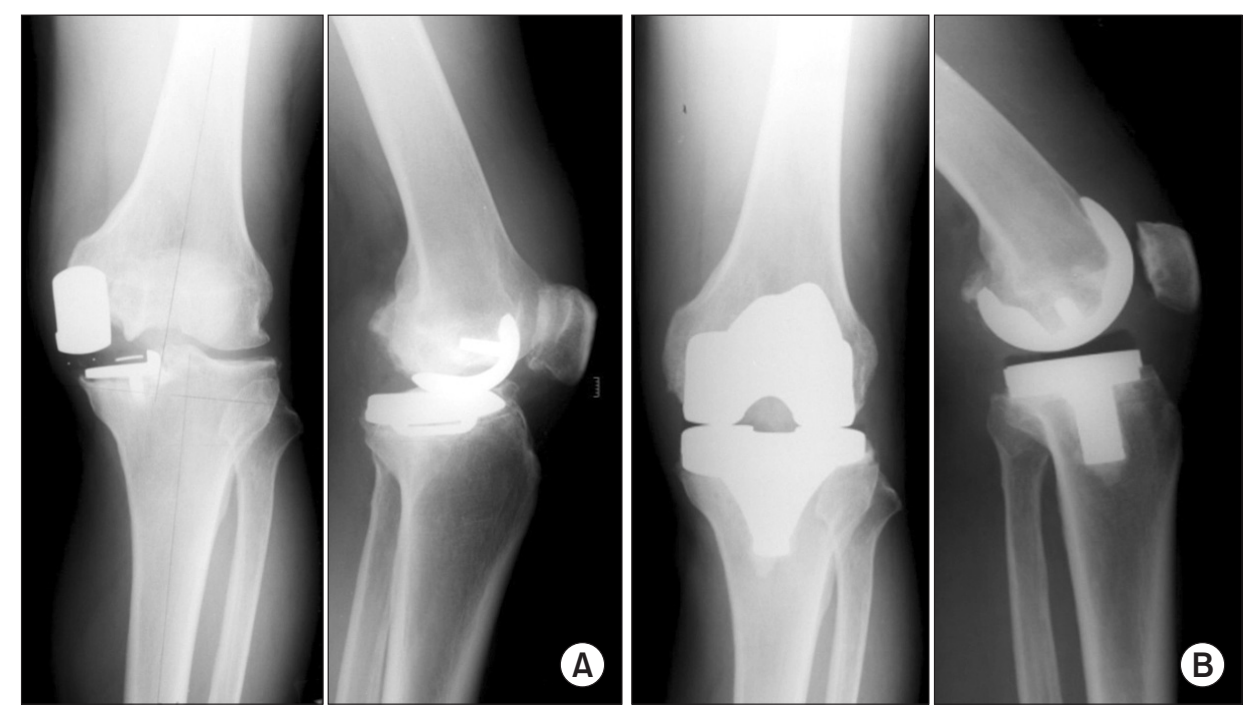

Fig. 1. (A) Radiographs of a 64-year-old male showing loosening of both femoral and tibial components at 3 years after unicompartmental knee arthroplasty. (B) Radiographs taken after conversion total knee arthroplasty using metal block.

Table 4. Treatment for Complications after Unicompartmental Knee Arthroplasty

\begin{tabular}{lcl}
\hline \multicolumn{1}{c}{ Reoperation procedure } & No. & \multicolumn{1}{c}{ Complication } \\
\hline Revision to TKA & 58 & 23 bearing dislocation, 22 loosening, 5 infection, 3 contralateral osteoarthritis, \\
& & $\begin{array}{l}\text { 2 tibial condylar fractures, 2 unknown origin pain, 1 polyethylene wear } \\
\text { Simple bearing change }\end{array}$ \\
Open reduction/internal fixation & 31 & 3 tibial condylar fractures \\
Arthroscopy & 2 & 2 impingement \\
MCL repair & 2 & 2 MCL rupture \\
Revision UKA & 1 & 1 loosening \\
Closed reduction/internal fixation & 1 & 1 femoral condylar fracture \\
Manipulation & 1 & 1 arthrofibrosis \\
Total & 89 & \\
\hline
\end{tabular}

TKA: total knee arthroplasty, MCL: medial collateral ligament, UKA: unicompartmental knee arthroplasty.

MCL repair $(n=2)$. In the 58 knees treated with TKA, the surgery was performed using an implant for primary TKA in 15 knees, whereas metal augmentation was required due to bone deficiency in the remaining 43 knees.

Regarding the treatment methods for each type of complication, bearing dislocation $(n=42)$ was treated either with simple bearing change $(n=24)$ or TKA $(n=18)$, and $5(21 \%)$ of the former 24 cases required conversion to TKA due to re-dislocation after bearing change. For the treatment of component loosening $(\mathrm{n}=23)$, TKA was carried out in 22 knees and revision UKA in 1 knee (Fig. 1). For polyethylene wear/destruction $(n=3)$, the bearing was changed in 2 knees and TKA was performed in 1 knee. For periprosthetic fracture $(n=5)$, open reduction and internal fixation was done in 4 knees with a tibial fracture, 1 of which later underwent TKA due to re-fracture, and TKA was the treatment of choice in the first place in 1 knee. Femoral fractures were treat- ed with closed reduction and internal fixation. MCL injury $(n=2)$ was repaired with suture. For the treatment of impingement in 2 knees, arthroscopic bone cement and osteophyte removal was performed. One knee with ankylosis was treated with manipulation under anesthesia. Progression of arthritis to the other compartment $(n=3)$ and unexplained pain $(n=2)$ were treated with TKA. All knees with infection $(n=5)$ underwent two-stage TKA for treatment (Table 4).

\section{Discussion}

In this single-center study, we reviewed the causes and types of complications $(n=89)$ that occurred following UKA $(n=1,576)$ and investigated optimal treatment methods. The incidence and type of complications were also compared among patients classified according to gender, medial/lateral UKA, and implant design 
and type. The strengths of this study include that continuous follow-up could be performed on a regular basis upon completion of surgery because all the patients were enrolled from the same institution and the sample size (UKA cases and complication cases) was relatively large compared to that in previous publications.

Complications that can occur following UKA include bearing dislocation, aseptic loosening, polyethylene wear, periprosthetic fracture, progression of arthritis to the contralateral compartment, infection, bone-implant impingement, retaining of cement debris in the joint, ankylosis of the knee, recurrent hematoma in the knee, and persistent pain ${ }^{2,10,11)}$.

Polyethylene insert dislocation is a major complication of mobile-bearing UKA that can occur in the presence of unbalanced flexion-extension gap, impingement of the bearing with adjacent bone or tibial/femoral component, instability of the medial compartment due to MCL injury, or secondary to femoral/tibial component loosening ${ }^{9,14)}$. For the treatment of bearing dislocation, bearing change, revision UKA, or conversion to TKA can be considered according to the cause of dislocation. In this study, 42 cases of bearing dislocation were noted and all of which occurred in the knees with the meniscal-bearing Oxford prosthesis, not in those with the tracked-bearing Preservation prosthesis. Of the 42 knees with bearing dislocation, 24 were treated with simple bearing change; however, re-dislocation occurred in $5(21 \%)$ after the treatment, which required conversion to TKA. Therefore, we suggest conversion TKA should be considered as an initial option in cases where the likelihood of redislocation after simple bearing change appears high based on the investigation of the cause of dislocation.

Factors that have been associated with early component loosening include younger age, overweight, thick polyethylene, varus deformity, and increased posterior tibial slope of the tibia. In particular, fixed-bearing UKA results in greater contact stress on the polyethylene insert due to low conformity, which may eventually lead to a failure associated with tibial component loosening or subsidence. And it is known that tibial component loosening is more often in the all-polyethylene prostheses than the metalbacked prostheses ${ }^{15-17)}$. Component loosening should be treated with a revision UKA or a conversion to TKA. In our study, component loosening was observed in 17 knees (1.2\%) with mobilebearing prostheses and in 6 knees (16.2\%) with fixed-bearing prostheses. The incidence of tibial component loosening was especially high in the knees with all-polyethylene fixed-bearing prosthesis. Conversion TKA was performed in 22 of these patients. In the remaining 1 knee with femoral component loosen- ing only, revision UKA involving polyethylene insert change was carried out.

Polyethylene wear is closely associated with the material of the insert used and the postoperative mechanical axis. In order to prevent wear/destruction of a polyethylene insert, UKA should be performed using a high quality polyethylene insert of sufficient thickness while taking care to avoid creating excessive varus alignment ${ }^{15,18)}$. If a failure occurs due to polyethylene wear in mobile-bearing or metal-backed fixed-bearing knees, simple insert change can be considered as a treatment method; however, conversion to TKA is the more common option in most cases. In our study, 3 cases of complication related to polyethylene wear were observed in the Oxford mobile-bearing prosthesis knees, and two of which were combined with polyethylene insert destruction. Although polyethylene wear was not observed in any of the knees with fixed-bearing prostheses in our study, this may be due to the length of the follow-up considering that the incidences were relatively high in previous studies. The mobile-bearing insert was changed in 2 knees and TKA was performed in 1 knee for the treatment of the complication.

Periprosthetic fractures following UKA are relatively rare and mostly observed around the tibial condyles unlike those after TKA. This can be attributed to the increased pressure and load applied on the proximal tibia, i.e., increased pressure per unit area ${ }^{11,15,19)}$. A tibal condylar fracture following UKA can be managed with conservative treatment if slight translation or varus deformity is present. If the fracture is accompanied by translation or deformity, open reduction and internal fixation should be performed. In the event of nonunion of the fracture, severe translation, or tibial component loosening, it needs to be revised to TKA $^{11}$. In our study, periprosthetic fractures were observed in 6 knees: 1 was medial femoral condylar fracture caused by trauma and the remaining 5 were medial tibial condylar fractures that occurred intraoperatively $(\mathrm{n}=1)$ or due to stress without trauma $(n=1)$ or trauma $(n=3)$. The tibial fractures were treated with TKA $(n=1)$ or open reduction and internal fixation $(n=5)$ but one of which was re-fractured requiring conversion to TKA. Femoral fractures were treated with open reduction and internal fixation under C-arm guided fluoroscopic control.

Progression of degenerative arthritis to the contralateral compartment is one of the major causes of failure following UKA ${ }^{2)}$. Overcorrection of the mechanical axis $\left(\geq 180^{\circ}\right.$ of valgus alignment) or progression of arthritis may cause degenerative changes in the lateral compartment ${ }^{18)}$. Progression of arthritis to the lateral compartment can be characterized by radiographic evidence of lateral joint space narrowing and osteophyte formation in 
the initial stage, which eventually leads to development of pain, subchondral sclerosis, and loss of joint space in the knee. In the current study, 3 out of 1,486 knees (0.2\%) with Oxford mobilebearing prosthesis showed progression of arthritis to the lateral compartment necessitating conversion to TKA. The incidence was significantly low comparted to that in the studies of Pandit et al. ${ }^{13)}$ and Epinette et al. ${ }^{3)}$, indicating that this complication may also be closely associated with lifestyle, sensitivity to pain, varus alignment of the knee joint, and the length of follow-up.

The MCL is the most import structure of the knee that determines the flexion-extension gap after UKA and significantly affects stability of the knee joint and implant. Therefore, the surgeon should take utmost care to protect the MCL during surgery and to secure bone-implant contact surface conformity to prevent chronic injury to the ligament ${ }^{2}$. Depending on the extent of damage, primary repair, augmentation, reconstruction of the ligament or conversion to TKA can be considered as a treatment option. In the current study, 2 cases of acute traumatic injury to the MCL following UKA were treated by primary repair. In some knees, overhang of the tibial component or use of a polyethylene insert of excessive thickness resulted in chronic MCL injury. Since most of these cases were combined with dislocation of the polyethylene insert, TKA was performed.

The risk of infection following UKA is significantly low compared to TKA because it can be performed through a relatively small incision allowing minimal joint exposure and bone resection and inflicts less damage to the adjacent soft tissue ${ }^{8}$. In the event of immediate or acute infection following UKA, early irrigation and debridement followed by antibiotic administration can be a proper treatment solution. However, if the initial treatment effort ends up in failure or chronic infection is present, the implanted prosthesis should be removed and a one-stage or twostage revision surgery should be carried out. In our study, the postoperative deep infection rate was remarkably low (5 cases, $0.3 \%)$. For the treatment of these knees, after open debridement and irrigation, antibiotic-impregnated bone cement was inserted, which was followed by two-stage TKA.

The incidence of ankylosis after UKA is lower than that after TKA because scar formation can be reduced during the minimally invasive procedure that causes lesser damage to the extensor mechanism and suprapatellar pouch ${ }^{15,20)}$. Postoperative ankylosis in UKA patients can be treated with manipulation under anesthesia, which often requires arthroscopic removal of intraarticular fibrous tissue and scar tissue. In our study, severe ankylosis (range, extension $10^{\circ}$ to flexion $80^{\circ}$ ) was observed in 1 patient in whom joint exercises were delayed due to pain. The patient could obtain satisfactory ROM (range, extension $0^{\circ}$ to flexion $130^{\circ}$ ) after manipulation under spinal anesthesia at 4 weeks after UKA.

Complications following UKA described in the literature are various in terms of the type and incidence according to the design and type of implant, follow-up period, and the surgeon who performed the surgery, but most of the reports are based on a small number of cases ${ }^{3,12-14,211}$. Pandit et al. ${ }^{13)}$ reported that the incidence of complications after phase 3 Oxford medial UKA was 29 knees $(2.9 \%)$ at 5.6 years after surgery. In the study, the common cause for revision surgery was progression of arthritis in the lateral compartment $(0.9 \%)$, dislocation of the bearing $(0.6 \%)$, and unexplained pain $(0.6 \%)$. In a study by Kim et al. ${ }^{21)}$ where the incidence of complications at a minimum 10-year follow-up was described as $9.6 \%$, the most prevalent complication was bearing dislocation (4.2\%) followed by component loosening (3.0\%). Epinette et al. ${ }^{3)}$ noted that component loosening (45\%) was the primary reason for failure after UKA, which was followed by progression of osteoarthritis (15\%) and polyethylene wear (12\%). In a study by Lim et al. ${ }^{12}$, , revision was required in $3.5 \%$ of the patients at a mean of 5.2 years after Oxford phase 3 UKA mostly due to bearing dislocation (3\%). Based on a review of 17 previous publications involving 3,138 Oxford phase 3 UKAs, Kim et al. ${ }^{14)}$ reported that the incidence of complications at a mean of 5-year follow-up was 146 knees (4.6\%). Their review described the primary cause for revision as bearing dislocation (47 knees, 1.5\%), which was found at least 3 times more prevalent among Asians than Westerners (3.0\% vs. $0.7 \%$ ). In our study, complications occurred in 89 (5.6\%) out of 1,576 knees after UKA in the following order of frequency: bearing dislocation (42 knees, 2.7\%), component loosening ( 23 knees, $1.5 \%$ ), periprosthetic fracture ( 6 knees, $0.4 \%$ ), infection ( 5 knees, $0.3 \%$ ), polyethylene wear ( 3 knees, $0.2 \%$ ), and progression of arthritis to the other compartment (3 knees, $0.2 \%$ ). Some authors attributed the higher prevalence of bearing dislocation among Asians including Koreans to the lifestyle where higher range of flexion is required during daily living activities. The results of our study were also in consistent with the previous studies: bearing dislocation was the most common complication among our patients (2.7\%) and the incidence was similar to that reported by Kim et al. ${ }^{14)}$ and Lim et al. ${ }^{12}$.

Peersman et al. ${ }^{22)}$ conducted a meta-analysis on the influence of the type and design of implants on postoperative complications by reviewing the outcomes of 4,330 fixed-bearing UKAs and 5,133 mobile-bearing UKAs. Major complications among fixed-bearing knees were progression of arthritis (0.29\%), component loosening $(0.22 \%)$, whereas those among mobile-bearing knees were component loosening $(0.44 \%)$, bearing dislocation $(0.29 \%)$, and 
progression of arthritis (0.23\%). In a meta-analysis, Ko et al. ${ }^{23)}$ found no difference in the frequency of complications between the fixed-bearing knees and mobile-bearing knees after UKA, but mobile bearing UKA patients were more predisposed to aseptic loosening, progression of arthritis, and bearing dislocation. In our patients, the incidence of complications was 82 knees (5.7\%) after the 1,441 mobile-bearing UKAs and 7 knees (5.1\%) after the 135 fixed-bearing UKAs. The most common complication was bearing dislocation (42 knees, $2.9 \%$ ) followed by component loosening (17 knees, 1.2\%) in the mobile-bearing knees, whereas component loosening (6 knees, 4.4\%) was most prevalent in the fixed-bearing knees and there was 1 knee with tibial condylar fracture.

Complications following UKA can be treated with revision operations or non-revision reoperations ${ }^{4,10)}$. Instead of a revision operation, incision and debridement, arthroscopic surgery, manipulation, ligament repair, or open reduction can be selected for treatment depending on the type of complication. In revision operations, simple polyethylene insert change, revision UKA, or conversion to TKA can be considered as an option. In a study by Clark et al. ${ }^{10)}$, complications occurred in $40(10 \%)$ out of 398 knees at a mean of 43 months after Oxford phase 3 UKA, and 15 knees (3.8\%) were treated with a revision surgery and the remaining 25 knees (6.2\%) underwent non-revision reoperations. Hamilton et al. ${ }^{4}$ found complications in 25 (11.3\%) out of 221 knees at a mean of 2 years after UKA using an all-polyethylene fixed-bearing Preservation prosthesis. They treated the complications with revision operations in 9 knees $(4.1 \%)$ and non-revision operations in the remaining 16 knees (7.2\%). In our study, the 89 cases of complications were treated with revision TKA $(n=58)$, revision UKA $(n=1)$, simple bearing change $(n=21)$, and nonrevision operations including arthroscopic surgery $(n=9)$.

Epinette et al. ${ }^{3)}$ retrospectively reviewed 418 cases of UKA performed at 25 different institutions. They found that complications occurred at a mean of 6.21 years (range, 0 to 24.7 years) after surgery: specifically, within 1 year after surgery in $19.1 \%$ and within 5 years after surgery in $48.5 \%$. A meta-analysis by Peersman et al. ${ }^{22)}$ showed that the mean interval from surgery to the development of complication was 6.0 years in the fixed-bearing knees and 2.4 years in the mobile-bearing knees. In the fixed-bearing knees, progression of arthritis to the other compartment was the most prevalent complication that occurred at a mean of 7.9 years after surgery and component loosening was noted at a mean of 7.7 years after surgery. In the mobile-bearing knees, component loosening and bearing dislocation occurred at a mean of 3.8 years and 2.0 years after surgery, respectively. In our patients, compli- cations following UKA were found at a mean of 4 years and 6 months postoperatively and $28.1 \%$ of which was noted within 1 year after surgery and $59.6 \%$ was observed within 5 years after surgery. The mean interval from UKA to the development of bearing dislocation, component loosening, polyethylene insert wear/destruction, and progression of arthritis to the other compartment was a 4 years and 4 months, 4 years and 10 months, 8 years and 4 months, and 11 years and 6 months, respectively.

We acknowledge some limitations of this study. First, the analysis on the postoperative complications was retrospective without any prospective research on the predisposing factors for the complications. Second, there were non-negligible differences between groups in terms of age and incidence of medial/lateral UKA. In addition, there was considerable disparity among prostheses with regard to the sample size and follow-up period, which could have affected the accuracy of comparisons. However, the focus of this research was not on the statistical comparisons but on the overall understating of the overall aspect of complications following UKA. Furthermore, we believe our in-depth analysis and explanation provides additional insight into the most commonly performed mobile-bearing UKA.

\section{Conclusions}

The most common complication following UKA was bearing dislocation in the mobile-bearing knees and component loosening in the fixed-bearing knees. The incidence of polyethylene wear and progression of arthritis to the other compartment was relatively low. The results of our study are in some discrepancy with those of studies involving Western patients. This can be attributed to the differences in patient characteristics such as lifestyle and in the type and design of implant used. Therefore, we believe that thorough understanding of UKA, proper patient selection, appropriate implant choice are essential to reduce complications following UKA and obtain satisfactory outcomes. We suggest that complications following UKA should be treated differently according to the type and cause of complication and conversion TKA can be the most effective treatment when revision operation is determined necessary.

\section{Conflict of Interest}

No potential conflict of interest relevant to this article was reported. 


\section{References}

1. Berger RA, Della Valle CJ. Unicompartmental knee arthroplasty: indications, techniques, and results. Instr Course Lect. 2010;59:47-56.

2. Vardi G, Strover AE. Early complications of unicompartmental knee replacement: the Droitwich experience. Knee. 2004;11:389-94.

3. Epinette JA, Brunschweiler B, Mertl P, Mole D, Cazenave A; French Society for Hip and Knee. Unicompartmental knee arthroplasty modes of failure: wear is not the main reason for failure: a multicentre study of 418 failed knees. Orthop Traumatol Surg Res. 2012;98(6 Suppl):S124-30.

4. Hamilton WG, Collier MB, Tarabee E, McAuley JP, Engh CA Jr, Engh GA. Incidence and reasons for reoperation after minimally invasive unicompartmental knee arthroplasty. J Arthroplasty. 2006;21(6 Suppl 2):98-107.

5. Lombardi AV Jr, Berend KR, Berend ME, Della Valle CJ, Engh GA, Fitz W, Hurst JM, Jinnah RH, Lonner JH, Macaulay WB, Repicci JA, Scuderi GR. Current controversies in partial knee arthroplasty. Instr Course Lect. 2012;61:347-81.

6. Lyons MC, MacDonald SJ, Somerville LE, Naudie DD, McCalden RW. Unicompartmental versus total knee arthroplasty database analysis: is there a winner? Clin Orthop Relat Res. 2012;470:84-90.

7. Morris MJ, Molli RG, Berend KR, Lombardi AV Jr. Mortality and perioperative complications after unicompartmental knee arthroplasty. Knee. 2013;20:218-20.

8. Goodfellow J, O'Connor, Dodd C, Murray D. Management of complications. In: Goodfellow J, O’Connor, Dodd C, Murray D, eds. Unicompartmental arthroplasty with the Oxford knee. Oxford: Goodfellow; 2011. p155-71.

9. Kim KT, Lee S, Park HS, Cho KH, Kim KS. A prospective analysis of Oxford phase 3 unicompartmental knee arthroplasty. Orthopedics. 2007;30(5 Suppl):15-8.

10. Clark M, Campbell DG, Kiss G, Dobson PJ, Lewis PL. Reintervention after mobile-bearing Oxford unicompartmental knee arthroplasty. Clin Orthop Relat Res. 2010;468:576-80.

11. Kim KT, Lee S, Cho KH, Kim KS. Fracture of the medial femoral condyle after unicompartmental knee arthroplasty. J Arthroplasty. 2009;24:1143.

12. Lim HC, Bae JH, Song SH, Kim SJ. Oxford phase 3 unicom- partmental knee replacement in Korean patients. J Bone Joint Surg Br. 2012;94:1071-6.

13. Pandit H, Jenkins C, Gill HS, Barker K, Dodd CA, Murray DW. Minimally invasive Oxford phase 3 unicompartmental knee replacement: results of 1000 cases. J Bone Joint Surg Br. 2011;93:198-204.

14. Kim SJ, Postigo R, Koo S, Kim JH. Causes of revision following Oxford phase 3 unicompartmental knee arthroplasty. Knee Surg Sports Traumatol Arthrosc. 2014;22:1895-901.

15. Collier MB, Eickmann TH, Sukezaki F, McAuley JP, Engh GA. Patient, implant, and alignment factors associated with revision of medial compartment unicondylar arthroplasty. J Arthroplasty. 2006;21(6 Suppl 2):108-15.

16. Price AJ, Waite JC, Svard U. Long-term clinical results of the medial Oxford unicompartmental knee arthroplasty. Clin Orthop Relat Res. 2005;(435):171-80.

17. Riebel GD, Werner FW, Ayers DC, Bromka J, Murray DG. Early failure of the femoral component in unicompartmental knee arthroplasty. J Arthroplasty. 1995;10:615-21.

18. Hernigou P, Deschamps G. Alignment influences wear in the knee after medial unicompartmental arthroplasty. Clin Orthop Relat Res. 2004;(423):161-5.

19. Yang KY, Yeo SJ, Lo NN. Stress fracture of the medial tibial plateau after minimally invasive unicompartmental knee arthroplasty: a report of 2 cases. J Arthroplasty. 2003;18:801-3.

20. Pandit H, Jenkins C, Barker K, Dodd CA, Murray DW. The Oxford medial unicompartmental knee replacement using a minimally-invasive approach. J Bone Joint Surg Br. 2006;88:54-60.

21. Kim KT, Lee S, Kim JH, Hong SW, Jung WS, Shin WS. The survivorship and clinical results of minimally invasive unicompartmental knee arthroplasty at 10-year follow-up. Clin Orthop Surg. 2015;7:199-206.

22. Peersman G, Stuyts B, Vandenlangenbergh T, Cartier P, Fennema P. Fixed- versus mobile-bearing UKA: a systematic review and meta-analysis. Knee Surg Sports Traumatol Arthrosc. 2015;23:3296-305.

23. Ko YB, Gujarathi MR, Oh KJ. Outcome of unicompartmental knee arthroplasty: a systematic review of comparative studies between fixed and mobile bearings focusing on complications. Knee Surg Relat Res. 2015;27:141-8. 